PROCEEDINGS OF THE

AMERICAN MATHEMATICAL SOCIETY

Volume 127, Number 7, Pages 2163-2165

S 0002-9939(99)04966-7

Article electronically published on March 17, 1999

\title{
ON THE PARTITION OF THE 2-SPHERE BY GEODESIC NETS
}

\author{
ALADÁR HEPPES
}

(Communicated by Christopher Croke)

\begin{abstract}
The main result of the paper is that for every natural number $n$ there exists a geodesic net with vertices of degree 3 or 4 partitioning the round 2 -sphere into $n$ regions.
\end{abstract}

\section{INTRODUCTION}

To answer a question of Poincaré it has been proved (see [Cr] and [Ha-Mo1]) that on any positively curved 2-sphere there exists a simple closed geodesic dividing the surface into two parts. In a recently published paper J. Hass and F. Morgan [Ha-Mo2] studied the existence of more complicated graphs that admit no length decreasing local perturbations (without changing the structure of the graph) on the surface of a sphere in different metrics. They define a geodesic net as a graph embedded on a surface that consists of geodesic arcs whose tangent unit vectors sum to zero at each vertex. Having shown the existence of certain geodesic nets that subdivide the surface into a few parts the authors formulated at the end of their paper the following

Conjecture. Let $S$ be a smooth Riemannian 2-sphere and $n$ a natural number. Then there is a geodesic net with vertices of degree 3 or 4 partitioning $S$ into $n$ regions.

(For $n \leq 4$ the claim of the conjecture follows from Theorems 1 and 2 of [Ha-Mo2].) It has also been mentioned that the answer is not known even in the case of the round sphere. We are going to deal with this special case and will show that the conjecture is true for the round sphere. Our proof is constructive.

Degree 3 nets. On the sphere the great circle is, up to congruency, the only closed geodesic, i.e. the only geodesic net without a vertex. It is also known [He] that there are 9 geodesic nets having just degree 3 vertices. As the above 10 nets divide the surface of the sphere into $2, \ldots, 10$ and 12 domains, it is practical to denote them by $N_{i}$, where $i$ refers to the number of pieces.

Received by the editors October 21, 1997.

1991 Mathematics Subject Classification. Primary 53C22; Secondary 53A10.

Key words and phrases. Geodesic, net, partition.

The author was partially supported by the Hungarian National Science Foundation.

(C)1999 American Mathematical Society 
Degree 4 nets. A geodesic net with just degree 4 vertices is necessarily the union of several great circles, i.e. $N_{2}$ nets. A set of $k$ great circles in general position (no three of them passing through a point) generates $2 k(k-1) / 2$ vertices and-by Euler's formula - the number of faces is $n=k(k-1)+2, k>0$.

Consequently, apart from these exceptional values listed so far, for $n>12$ the presence of both degree 3 and degree 4 vertices is necessary.

Construction strategy. The partition of the sphere into $n$ domains - in the cases not covered by the 10 isogonal nets - can be done superimposing a single degree 3 net and several great circles. In the constructions below - to avoid vertices of degree higher than 4 -it is assumed (without repeating it) that the vertex of a component does not lie on an edge of another component.

The union of an $N_{5}$ or $N_{7}$ net and a great circle. We remind the reader that for $i=5$ and 7 the isogonal net $N_{i}$ is the central projection of the one-skeleton of a regular $(i-2)$-gonal pyramid of suitable size inscribed into the sphere. Assume that the $i$-gons are centered at the poles and let $C$ be a point on the equator in general position, i.e. not coplanar with any pair of vertices of $N_{i}$. Then a great circle through $C$ can contain at most one of the vertices. When the center of such a great circle is sufficiently close to the equator, it cuts 4 of the edges of the net: it crosses the top and bottom $(i-2)$-gons and two side faces. Moving the center continuously away from the equator will tilt the plane that will separate the vertices of the upper $(i-2)$-gon from their mirror images reflected in the equatorial plane one by one.

For $i=5$ after the first such separation the great circle is cutting 1 side edge, i.e. 1 more face ( 5 faces in total), and, if the center is sufficiently close to a pole, the number of intersections is 3 (only side faces are met). Thus for $i=5$ this cutting number can be 3,4 or 5 .

For $i=7$ if the great circle is tangent to the incircle of the pentagonal face (or close to this position) the number of intersections is 7 (all faces are cut), and a continuity argument shows that all values between 4 and 7 are also possible. Thus for $i=7$ there are great circles cutting the net $N_{7}$ in $4,5,6$ or 7 points.

The union of $N_{7}$ and several great circles. In view of the above discussion the total number of degree 4 intersections of an $N_{7}$ net with $k$ great circles can be any number between $4 k$ and $7 k$, while the number of additional (degree 4 ) vertices generated by the great circles among themselves is $k(k-1)$. Each new intersection means cutting a face of a net into two, i.e. increase the number of faces by 1 . Thus this number of new vertices has to be added to the original 7. As $k$ increases, the length $3 k+1$ of the covered intervals grows faster then their lower end $\left(k^{2}+3 k+7\right)$ does, and, from $k=3$ (from $n=25$ ) upward, all cases are settled. Even below this value (between 11 and 24) only three values, $n=15,16$ and 24 , are not covered by this set of constructions.

The union of $N_{5}$ and several great circles. The analogous construction based on $N_{5}$ results lower bound $3 k$ and upper bound $5 k$ for the number of intersections between the $k$ great circles and $N_{5}$; thus the total number of faces will be between $k^{2}+2 k+5$ and $k^{2}+4 k+5$. Consequently, the intervals for $k=2$ and $k=3$ cover the remaining cases.

This settles the Hass-Morgan conjecture for the round sphere. 
Remarks. 1) Although the above constructions admit a lot of freedom of choice on one hand in the exact position of the components, and on the other hand in choosing different combinations of the superimposed isogonal nets, it is evident that the angles at the degree 4 vertices get sharper and sharper as $n$ grows. It is natural to ask whether this is necessary, or an additional requirement for a positive lower bound for the angles could also be fulfilled.

2) The given constructions are unions of several simpler nets of type $N_{2}$ (with no vertex) and $N_{5}$ or $N_{7}$ with degree 3 vertices while the degree 4 vertices of the union are the points where the edges of the components meet. The fact that there exist infinitely many geodesic nets (having only degree 3 and degree 4 vertices) that are irreducible (i.e. not the union of simpler geodesic nets) raises the question if the partition of the sphere into $n$ domains is possible with irreducible geodesic nets of degree 3 and degree 4 vertices. As an example for an infinite set of irreducible geodesic nets having degree 4 vertices as well consider the vertices of a regular $(2 k+1)$-gon centered at the north pole and connect the vertices to get a regular star polygon. For suitable size of the $(2 k+1)$-gon the angles will be $2 \pi / 3$. This star polygon, its reflection in the equator plane and the $2 k+1$ "vertical" edges connecting the two polygons provide an irreducible geodesic net with $4 k+2$ vertices of degree 3.

\section{REFERENCES}

[Cr] C. Croke, Poincaré's problem on the shortest closed geodesic on a convex hypersurface, J. Diff. Geom. 17 (1982), 595-634. MR 84f:58034

[Ha-Mo1] J. Hass and Frank Morgan, Geodesics and soap bubbles on surfaces, Math. Z. 223 (1996), no. 2, 185-196. MR 97j:53009

[Ha-Mo2] J. Hass and Frank Morgan, Geodesic nets on the 2-sphere, Proc. of the AMS 124/12 (1996), 3843-385. MR 97b:53042

[He] A. Heppes, Isogonale sphärische Netze, Ann. Univ. Sci. Budapest Eötvös, Sect. Math. 7 (1964), 41-48. MR 30:3406

VÉrcse u. 24/A, H-1124 Budapest, Hungary

E-mail address: h9202hep@helka.iif.hu 